\title{
Comparison between SVM and KNN classifiers for iris recognition using a new unsupervised neural approach in segmentation
}

\author{
Hicham Ohmaid $^{1}$, S. Eddarouich ${ }^{2}$, A. Bourouhou ${ }^{3}$, M. Timouyas ${ }^{4}$ \\ ${ }^{1}$ National School of Computer Science and Systems Analysis (ENSIAS), Mohammed V University, Rabat/10112, \\ Morocco \\ ${ }^{2}$ Regional Educational Center, Rabat/10112, Morocco \\ ${ }^{3,4}$ Higher Normal School of Technical Education (ENSET), Mohammed V University, ENSET, Rabat/10112, Morocco
}

\begin{tabular}{|c|c|}
\hline Article Info & ABSTRACT \\
\hline Article history: & A biometric system of identification and authentication provides automatic \\
\hline Received Dec 12, 2019 & recognition of an individual based on certain unique features or \\
\hline Revised Feb 17, & dentification method that applies pattern recognition to images of the iris. \\
\hline Accepted Mar 19, 2020 & $\begin{array}{l}\text { Owing to the unique epigenetic patterns of the iris, iris recognition is } \\
\text { considered one of the most accurate methods in the field of biometric }\end{array}$ \\
\hline Keywords: & $\begin{array}{l}\text { identification. The segmentation algorithm proposed in this article starts with } \\
\text { determining the regions of the eye using unsupervised neural approach, after }\end{array}$ \\
\hline Biometric & the outline of the eye is found using the Canny edge, The Hough Transform \\
\hline Discrete w & $\begin{array}{l}\text { loyed to determine the center and radius of the pupil and the iris. Then } \\
\text { rmalization allows transforming the seomented circular iris region into }\end{array}$ \\
\hline Hough transform & lar shape using Daugman's rubber sheet model. A \\
\hline Iris segmentation & discrete wavelet transformation (DWT) is applied to the normalized iris to \\
\hline K-nearest-neighbor (KNN) & lower the size of iris models and to improve classifier accuracy. Finally, the \\
\hline Support vector machine (SVM) & URIBIS iris database is used for individual user verification by using the \\
\hline kernel function & upport vector machine (SVM) which based on the \\
\hline Unsupervised neural approa & \\
\hline
\end{tabular}

This is an open access article under the CC BY-SA license.

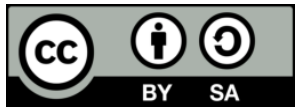

\section{Corresponding Author:}

Hicham Ohmaid,

National School of Computer Science and Systems Analysis (ENSIAS),

Mohammed V University, Rabat/10112, Morocco.

Email: hicham.ohmaid@gmail.com

\section{INTRODUCTION}

Nowadays, biometric recognition has become a reliable way to identify and recognize individuals. Conventional authentication methods like passwords and identification cards are not always credible because these methods can be forgotten or stolen. Identification based on physiological or behavioral characteristics of people (such as irises, fingerprints, faces, hand geometry, signatures, fingerprints, et cetera) can accurately authenticate people's identity [1].

Iris recognition offers the highest accuracy in identifying individuals compared to any other biometric approaches. The iris is defined as the circular part between the pupil and sclera of the eye. It is so unique that no two irises are alike, even among identical twins or between the left and right eye of the same person [2-3]. These visible characteristics are thought to be discriminatory and unique to each eye, as well as stable over an individual's lifetime. This makes the iris a very useful biometric identifier when it is possible to capture iris images of sufficient quality [4]. 
The system is inspired by Daugman's work [5]. It is composed of a number of sub-systems, which are described in Figure 1 and Figure 2 the contains stages of an iris detection system.

The segmentation step enables the localization of the iris region in an eye image, which consists of finding two contours of the iris (pupil and iris). In other words, the internal contour (pupil/iris) and outer (iris/sclera) are located. Once the iris region is segmented, the next stage is to normalize this segmentation. The normalization transforms the iris texture from Cartesian to polar coordinates in order to limit the problems of variations in the eye (like the optical size of the iris or the position of the pupil in the iris). This step is based on Daugman's rubber sheet model [5]. Feature extraction is a technique used to extract information from the iris image, in order to lower the size of iris models and improve classifier accuracy. These features can not be used to reconstruct images, but these values are used in classification. Finally, matching is performed by comparing the features of a template iris with the feature vectors of templates in the database, and a decision is formulated [6-7].

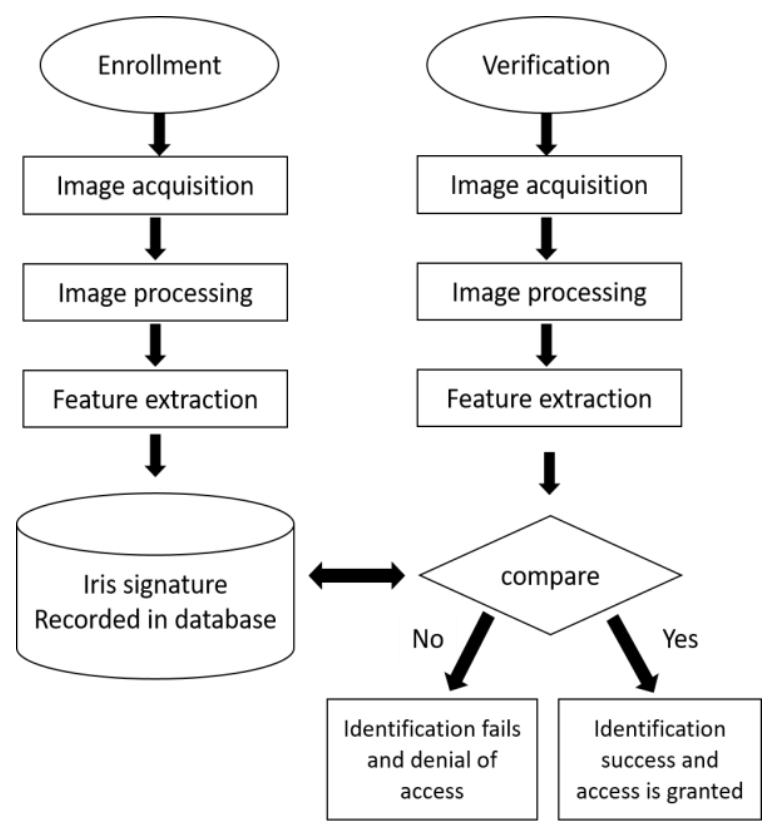

Figure 1. Iris recognition system

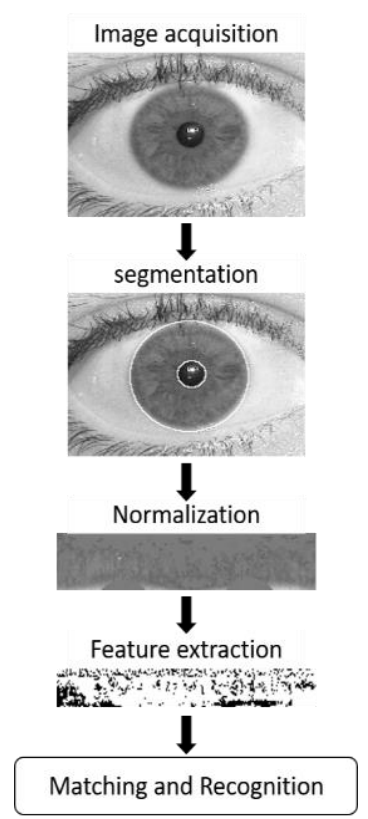

Figure 2. Typical stages of iris recognition

The main motivation in this research is to propose a new effective and robust algorithm to segment and classification the clear or noisy iris images. The proposed algorithm added a new pre-processing step using a new unsupervised neural approach to divide the iris image in two regions namely iris and eyelashes region, sclera and skin, in order to facilitate the determination of the iris contour in the phase Canny edge detection [8]. Unlike other iris segmentation algorithms, that process the whole image of the eye (which contains the non-iris regions that generate segmentation errors) such as Daugman algorithms. Then, discrete wavelet transformation (DWT) is used for extracting the optimum features of iris images and reducing the runtime of classification of these iris templates. Finally, a comparison was made between the classifiers SVM and KNN.

\section{METHODOLOGY}

The iris recognition system consists of the following steps:

- Image acquisition

- $\quad$ Iris segmentation

- Normalization

- Feature extraction

- Classification

In the approach presented here, segmentation was achieved using the Hough transform with the aim of locating the iris region of an eye image. The normalization enabled the transformation of the segmented 
circular iris region into a fixed-size rectangular shape using Daugman's rubber sheet model. Two level discrete wavelet transformation (DWT) was applied to the normalized iris for feature extraction. The support vector machine was used to classify the similarity between the iris templates. Figure 3 shows a diagram of the iris recognition system.

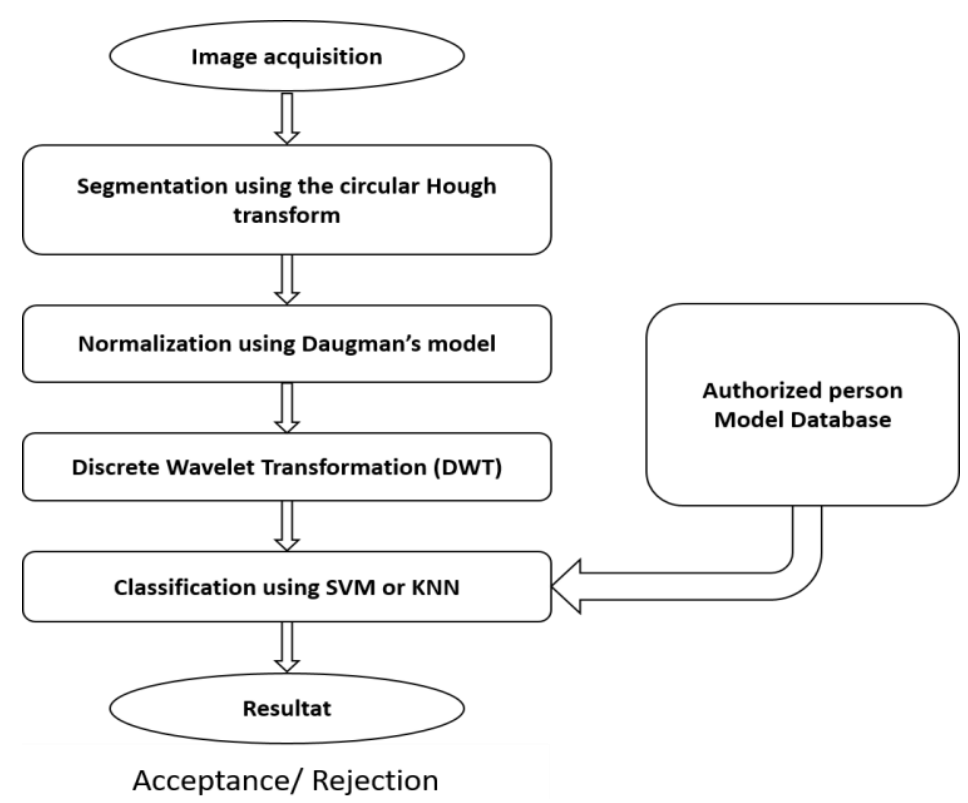

Figure 3. Diagram of iris recognition system

\subsection{Iris segmentation}

In this section, we present a new iris segmentation approach used as a pre-processing step, based on neural competitive concepts [9]. As shown in Figure 4, this approach allows partitioning the iris image in two regions namely iris and eyelashes region, sclera and skin. Then, the outline of the eye is found using the Canny edge, The Hough Transform is employed to determine the center and radius of the pupil and the iris. Figure 4 shows the iris segmentation steps.

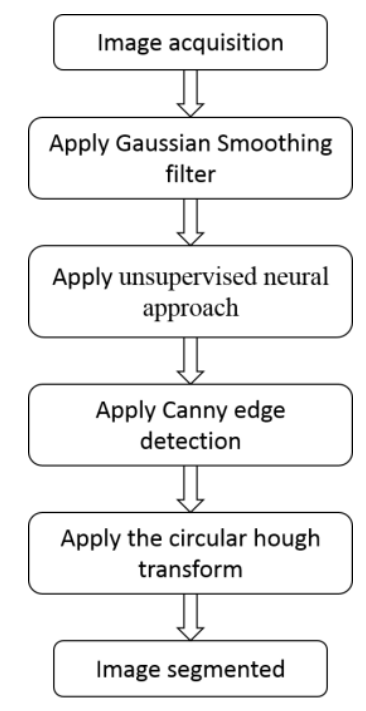

Figure 4. Algorithm used for iris recognition 


\subsubsection{Iris segmentation using the unsupervised neural approach}

In an unsupervised context, that means when no prior information about the data sample, we involve the neural competitive clustering procedure to the Iris segmentation. The objective of these approaches to classifying the image pixels according to their distribution in the representation space and to assign them a label. As illustrated in Figure 5, it starts first by the organizationIt starts first, by the organization of the pixels of Iris image in an observation matrix (each row represents a pixel and each column represents an attribute) to estimate the underlying probability density function (pdf)of the pixels distribution using a non-parametric estimator. In the second step, the procedure uses an artificial neural network with competitive training (NNCT) to extract the local maxima of the pdf. Following a modes detection method using a technique to detect the existing interneural connection [10]. The last step is for affecting the remaining pixels to their classes.

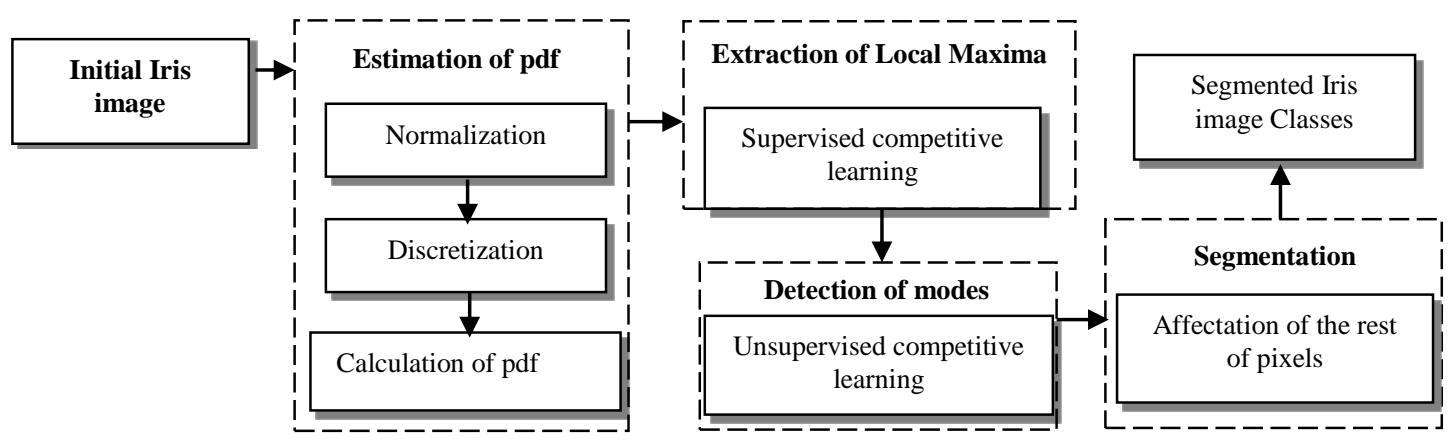

Figure 5. Architecture of the segmentation procedure

\subsubsection{The estimation of underlying probability density function}

After constructing the observation matrix of an Iris image pixels $\Gamma=\left\{X_{1}, X_{2}, \ldots, X_{Q}\right\}$ considering as a set of $\mathrm{Q} \mathrm{N}$-dimensional observations (in this case $\mathrm{N}=3$ ) With $\mathrm{X}_{q}=\left[x_{q, 1}, x_{q, 2}, \ldots, x_{q, n}, \ldots, x_{q, N}\right], \mathrm{q}=1,2, \ldots, \mathrm{Q}$ and a probability density function $\mathrm{P}(\mathrm{X})$, anon-parametric method based on The Parzen [11] window using to estimate this underlying density function. The technique is a fast estimation algorithm that is proposed by Postaire and Vasseur [12]. First, the range of variation of each component of these observations is normalized to the interval $[0, \mathrm{R}]$, where $\mathrm{R}$ is an integer such as $R \geq 2$, by means of the transformation defined as:

$$
y_{n, q}=\frac{\left(x_{n, q}-\min _{q} x_{n, q}\right)}{\left(\max _{q} x_{n, q}-\min _{q} x_{n, q}\right)} * R
$$

Each axis of the so normalized data space is then partitioned into $\mathrm{R}$ exclusive intervals of unit width. This discretization defines a set of $R^{N}$ hypercube of unit side length. Each hypercube noted $H(X)$, is a site defined by its $\mathrm{N}$ coordinates $x_{1}, x_{2}, \ldots, x_{n}, \ldots, x_{N}$ which are the integer parts of the coordinates of its centre $X$. To be more specific, let $Y_{q}=\left[y_{1, q}, y_{2, q}, \ldots, y_{n, q}, \ldots, y_{N, q}\right], \mathrm{q}=1,2$, Q be the Q observations in the normalized space. Each observation $\mathrm{Yq}$ is found inside a non-empty hypercube with the coordinates $x_{n}=\operatorname{int}\left(y_{n, q}\right), \mathrm{n}=1,2, \mathrm{~N}$, where $\operatorname{int}\left(y_{n, q}\right)$ designates the integer parts of $y_{n, q}$. If several observations fall in the same hypercube, this one appears many times on the list of non-empty hypercubes. Furthermore, the number of times the hypercube $\mathrm{H}(\mathrm{X})$ appears in that list indicates the number of data points $\mathrm{q}[\mathrm{H}(\mathrm{X})]$ which falls in this hypercube. Subsequently, the value of the local density estimated is:

$$
p(X)=\frac{q[H(X)]}{Q}
$$

Since the volume of $\mathrm{H}(\mathrm{X})$ is equal to unity.

So, this fast procedure allows only the estimation of the underlying probability density function at the centers of the non-empty hypercube whose number never exceeds the number Q of available observations (pixels). At the centers of the hypercube cells, which are not on that list, the density estimates are known to be null. At the end of this fast algorithm, all the available information for clustering is in the discrete set $\underline{X}$ of estimated values of the underlying probability density function $P(X)$. 


\subsubsection{The extraction of local maxima by neural network}

Assimilating the modes to the local maxima of the pdf, the proposed approach uses the Neural Networks with Competitive Training (NNCT) [13]. In the training algorithm, we work only on the pdf by presenting sequentially, the centers of the non-empty hypercube of the set $\mathrm{X}$ to the network, instead of the Q observations.

The neural network is composed of two layers: the input layer and the output layer. The first one is made of $\mathrm{N}$ units $I_{n}, \mathrm{n}=1,2, \ldots, \mathrm{N}$, such that unit $I_{n}$ is solicited by the attribute $\mathrm{X}_{\mathrm{N}}$ of the non-empty hypercube $\mathrm{H}(\mathrm{X})$ when this one is presented to the network. However, each output neuron materializes a hypercube which represents the site of one local maximum of the pdf in the set $\mathrm{X}$, and presents its weight by the mean vector $\mu_{k}(X), \mathrm{k}=1,2, \ldots, \mathrm{K}$. The number of the output neuron is first initialized arbitrarily.

During the training phase, the output neurons enter into competition with each other by comparing the distance $D\left[\mu_{k}(X), H(X)\right], \mathrm{k}=1,2, \ldots, \mathrm{K}$, between the input hypercube $\mathrm{H}(\mathrm{X})$ and each output neuron $\mu_{k}(X)$, the winner is the closest one to the hypercube, then we compare the values of the pdf associated to the winner neuron $\mu_{g}(X)$ and to $\mathrm{H}(\mathrm{X})$. The distance measure used in this training algorithm is Mahalanobis distance that gives the best results for the non-Gaussian distribution [14] instead of Euclidian distance as in the (NNCT).

\subsubsection{Detection of significant modes of pdf}

The aim of this phase is to connect each group of the closest modes in such a way that we get a map which preserves the shape and structure of the classes existing in the image, by an improved Competitive Hebbian Learning method (CHLim) [10] which allows eliminating the influence of the output neural number.

To generate the induced Delaunay triangulation, the CHLim, given the K modes detected by CNN as prototypes in RN, successively adds connections among them. The method does not change the weight of prototypes, but only generates topology according to these prototypes. For each mode $\mathrm{H}\left(X_{k}\right)$, can be connected to the two closest prototypes by an edge using Mahalanobis distance as a measure of resemblance instead of Euclidian distance, it works as an activation function for competition between neurons. This leads to the induced Delaunay triangulation, which is limited to those regions of the input space $R^{N}$.

\subsubsection{Classification procedure}

Once the different modes are identified, the classification method [15] that we use in the present work consists first, in defining the pixels falling into any mode of a connected set as the prototype of one cluster. Then, the remaining pixels, which do not fall in one of the detected modes, are assigned to the clusters attached to their nearest neighbor among these prototypes by means of Mahalanobis distance. Figure 6 presents the procedure for classifying the iris image.

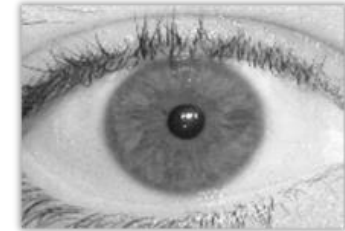

(a) Original image

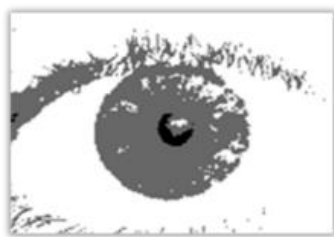

(f) Segmented image

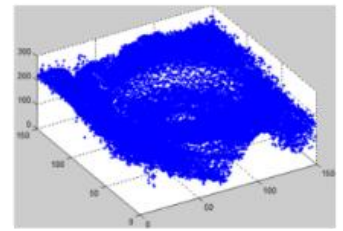

(b) Pxels in tridimensional space

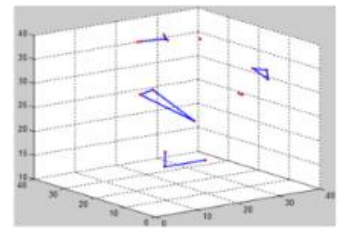

(e) 6 modes detected

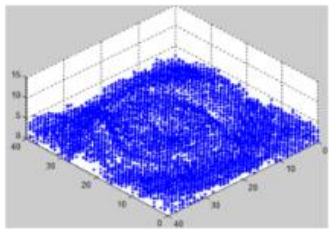

(c) Estmation of the underlying pdf

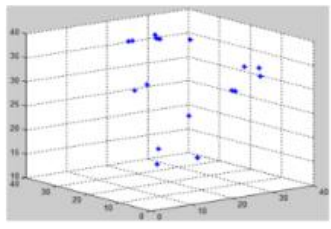

(d) 17 maxima extracted after 4 epoch

Figure 6. Illustration of the Iris segmentation procedure

\subsubsection{Hough transform}

Hough transform is a standard image analysis tool for finding curves that can be defined in a parametrical form such as lines, circles, parabolas, and hyperbolas [16]. The edge map is then used in a 
voting process to maximize the defined Hough transform for the desired contour. Considering the obtained edge points as $(x j ; y j), j=1 ; 2$; n, a Hough transform can be written as:

$$
H\left(x_{c}, y_{c}, r\right)=\sum_{j=1}^{n} h\left(x_{j}, y_{j}, x_{c}, y_{c}, r\right)
$$

Where

$$
h\left(x_{j}, y_{j}, x_{c}, y_{c}, r\right)=\left\{\begin{array}{c}
1 \text { if } g\left(x_{j}, y_{j}, x_{c}, y_{c}, r\right)=0 \\
0 \text { otherwise }
\end{array}\right.
$$

The limbus and pupil are both modeled as circles and the parametric function $\mathrm{g}$ is defined as:

$$
g\left(x_{j}, y_{j}, x_{c}, y_{c}, r\right)=\left(x_{j}-x_{c}\right)^{2}+\left(y_{j}-x_{c}\right)^{2}-r^{2}
$$

Assuming a circle with center (xc; yc) and radius $\mathrm{r}$, the edge points that are located over the circle result in a zero value for the function. The value of $g$ is then transformed to 1 by the $h$ function, which represents the local pattern of the contour. The local patterns are then used in a voting procedure using the Hough transform, $\mathrm{H}$, in order to locate the proper pupil and limbus boundaries. In order to detect the limbus, only vertical edge information is used. The two eyelids usually cover the upper and lower parts; these parts contain horizontal edge information. The horizontal edge information is used for detecting the upper and lower eyelids, which are modeled as parabolic arcs [9].

After the Hough Transform process is complete, six parameters are stored: the radius and $\mathrm{x}$ and $\mathrm{y}$ (the center coordinates for both circles). Eyelids are isolated by first fitting a line to the upper and lower eyelid using the linear Hough transform. A second horizontal line is then drawn that intersects with the first line at the iris edge that is closest to the pupil. This process is completed for both the top and bottom eyelids. The second horizontal line allows maximum isolation of eyelid regions. Figure 7 shows an example of properly segmented iris.

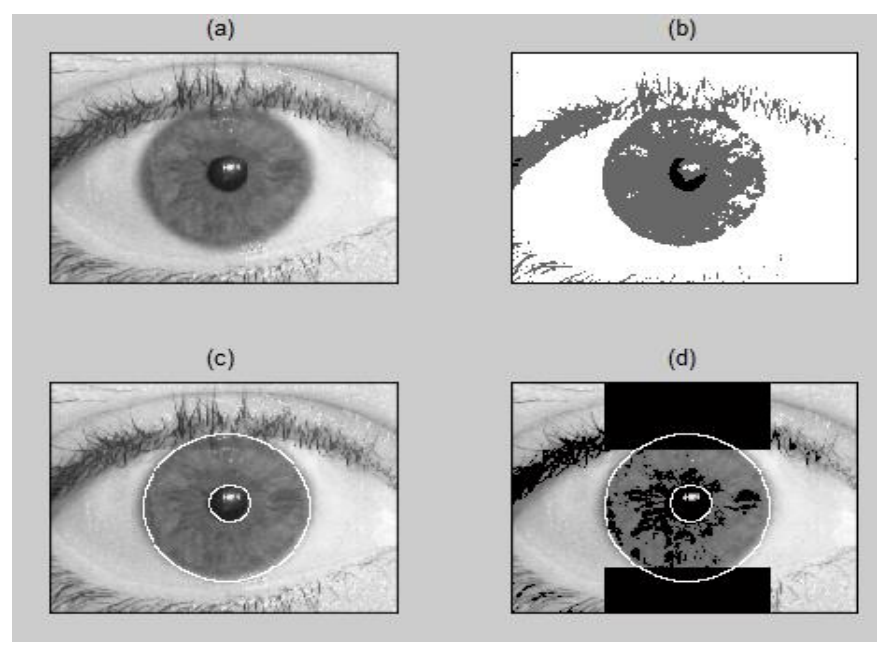

Figure 7. Examples of the segmented iris

\subsection{Iris normalization}

The size of the iris may change because of variation in the illumination, even for an iris from the same person. This elastic deformation in iris texture must be compensated for to achieve more accurate recognition results. Normalization is a technique to prepare a segmented iris for feature extraction and transforms the segmented circular iris region into a fixed-size rectangular shape. The Cartesian-to-polar transform of the iris region is based on Daugman's rubber sheet model [5], as illustrated in Figure 8. 


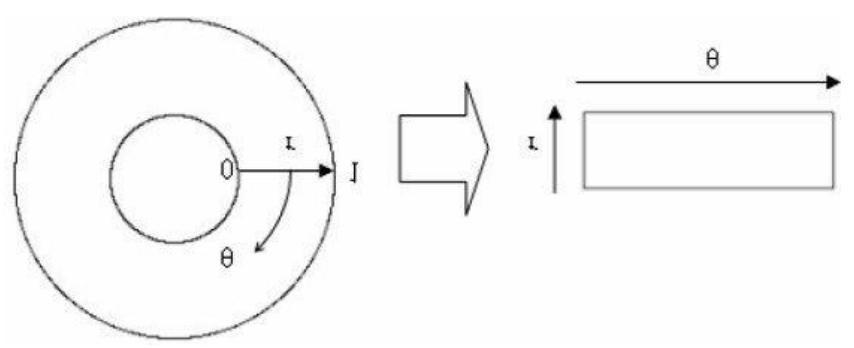

Figure 8. Cartesian-to-polar coordinate transform

The Cartesian-to-polar transform of the iris region is modeled by (4).

$$
I(x(r, \theta)), y(r, \theta)) \rightarrow I(r, \theta)
$$

with

$$
\begin{aligned}
& x(r, \theta)=(1-r) x_{p}(\theta)+r x_{i}(\theta) \\
& y(r, \theta)=(1-r) y_{p}(\theta)+r y_{i}(\theta)
\end{aligned}
$$

The coordinates of the inner and outer boundaries along the $\theta$ direction are $\mathrm{xp}$, yp and $\mathrm{xi}$, yi, respectively. Image-normalized enhancement is required to obtain accurate features because the normalized iris presents many variations due to variations in light illuminations. By using histogram equalization, pixels intensities are spread over the entire iris template. Figure 9 shows an iris template with histogram equalization.

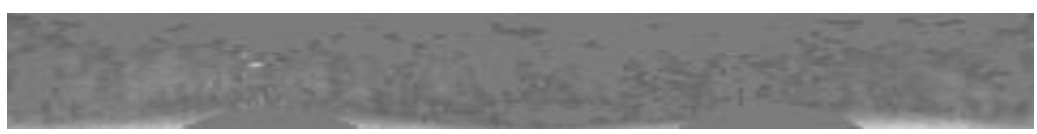

(a)

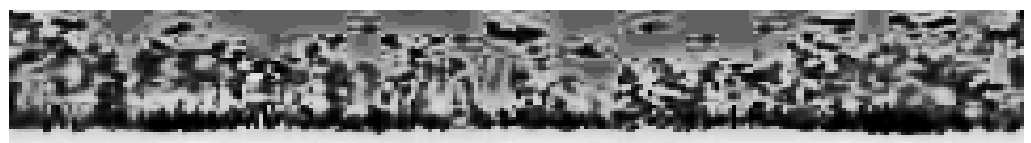

(b)

Figure 9. (a) Iris normalized into polar coordinates, (b) Enhancement of the iris normalized image

\subsection{Feature extraction}

In the encoding stage, two level discrete wavelet transformation (DWT) is applied to the normalized iris region, in order to determine DWT coefficients, by passing the signal in different frequency ranges, namely low-low (LL), low-high (LH), high-low (HL) and high-high (HH), as indicated in Figure 10. The frequency range can be represented as LL $<$ LH $<$ HL $<$ HH [17-18].

The LL sub-band represents the feature characteristics of the iris; this sub-band (LL) is used in the classification phase. Figure 11 indicates the resolution of the normalized and enhanced iris image (48x432). After applying DWT to an enhanced iris image, the resolution of sub-band is $(24 \times 216)$. This sub-band is used instead of the original normalized iris data to reduce the resolution of the iris model, and the runtime of the classification is similarly reduced. 


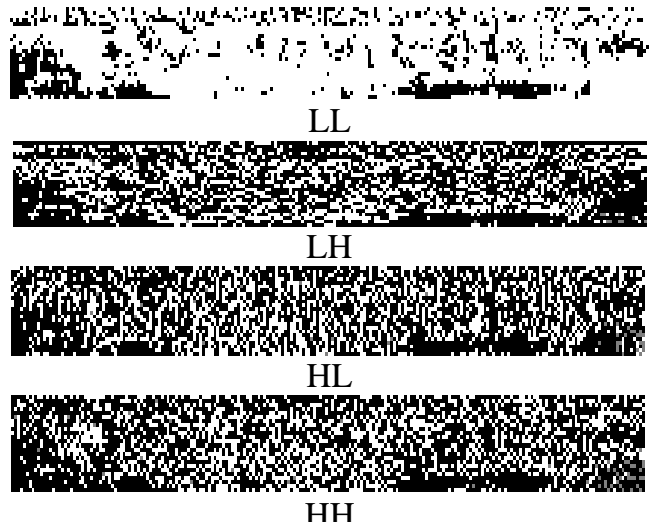

$\mathrm{HH}$

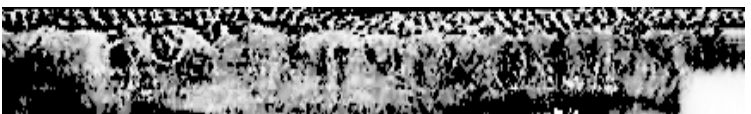

(a)

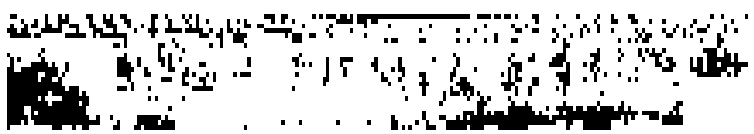

(b)

Figure 11. (a) Enhancement of the iris normalized image, (b) DWT transforms normalized iris image into LL sub-bands

Figure 10. Iris template using DWT

\subsection{Support vector machine SVM}

The support vector machine (SVM) performs pattern recognition according to the principle of structural risk minimization. The development of SVM as a classifier has two major aspects: the first aspect is to find the hyperplane that optimally separates the two classes, and the second aspect is the transformation of the linearly non-separable classification problem into a linearly separable problem [19].

Given an input entity vector learning set and the class tag $\left(\mathrm{x}_{-} \mathrm{i}, \mathrm{y} \_\mathrm{i}\right), \mathrm{i}=1,2 \ldots, 1$, where $x_{i} \in R^{n}$ and $y \in\{1,-1\}$. In the case of a linearly separable problem, the separating hyperplane (which defines the boundary between class 1 and class 2) can be represented as follows:

$$
\min _{\omega, b, \xi} \frac{1}{2} \omega^{T} \omega+C \sum_{i=1}^{l} \xi_{i}
$$

Subject to

$$
y_{i}\left(\omega^{T} \phi\left(x_{i}\right)+b\right) \geq 1-\xi_{i} ; \xi_{i} \geq 0
$$

In the case of a non-linearly separable problem, the training vectors $\mathrm{x} \_\mathrm{i}$ are mapped into higher dimensional space by the function $\varnothing$ in order to make the problem linearly separable. The SVM then finds a linear separating hyperplane with the maximal margin in this higher dimensional space. The penalty parameter of the error term is C > 0 . Furthermore, $K\left(x_{i}, x_{j}\right)=\emptyset\left(x_{i}\right)^{T}\left(x_{j}\right)$ is called the kernel function [20].

- Linear:

$$
K\left(x_{i}, x_{j}\right)=x_{i}^{T} x_{i}
$$

- Polynomial:

$$
K\left(x_{i}, x_{j}\right)=\left(\gamma x_{i}^{T} x_{j}+r\right)^{d}, \gamma>0
$$

- Gaussian:

$$
K\left(x_{i}, x_{j}\right)=\exp \left(-\frac{\left\|x_{i}-x_{j}\right\|^{2}}{2 \gamma^{2}}\right) \gamma>0
$$

\section{RESULTS}

The Classification Learner App (included in the Statistics and Machine Learning Toolbox for MATLAB) is an application that can be used to train models to classify data using supervised machine learning methods. The SVM and KNN classifiers were used for our approach [20-21]. 
By using Classification Learner, we can explore data, select features, specify validation schemes, and train and export classification models to the MATLAB workspace to generate predictions for new data.

To evaluate the effectiveness of the proposed system, a set of eye images obtained from the UBIRIS databases were used [22]. Figure 12 shows an example of the eye images contained in this database. The total number of iris classes is 100 , and each iris class contains three images stored in the database for this experiment. In this work, 300 images were used for training, and 100 images were used for testing purposes.

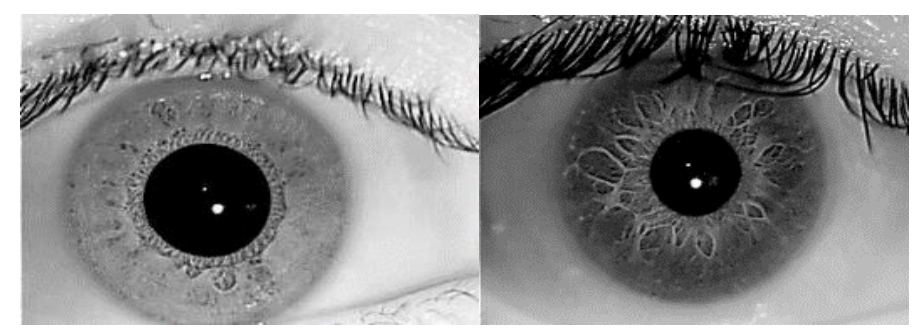

Figure 12. Eye images in the UBIRIS database

Four kernel functions (Linear, Quadratic, Cubic, and Medium Gaussian) were used, and the classification efficiency of each kernel was measured. Since the highest classification accuracy was obtained by a Medium Gaussian kernel, as shown in Table 1, this kernel was used in our system for classification and recognition purposes. As the simulation result shown in Table 2, the KNN algorithm assured better the accuracy of $95 \%$, as well as the execution time is too low compared to that of the SVM algorithm.

Table 1. Efficiency of various kernel functions (SVM)

\begin{tabular}{ccc}
\multicolumn{3}{c}{ functions (SVM) } \\
\hline Kernel Type & $\begin{array}{c}\text { Classification } \\
\text { Accuracy }(\%)\end{array}$ & $\begin{array}{c}\text { Training Time } \\
(\mathrm{sec})\end{array}$ \\
\hline Linear & 68.2 & 430.89 \\
Quadratic & 84.3 & 533.09 \\
Cubic & 88.6 & 580.83 \\
Medium Gaussian & 88.9 & 602.28 \\
\hline
\end{tabular}

Table 2. Efficiency of KNN classifier

\begin{tabular}{ccc}
\hline & $\begin{array}{c}\text { Classification Accuracy } \\
(\%)\end{array}$ & $\begin{array}{c}\text { Training Time } \\
(\mathrm{sec})\end{array}$ \\
\hline $\mathrm{KNN}$ & 95.00 & 19.94 \\
\hline
\end{tabular}

The accuracy of our proposed technique is $95 \%$, is shown in Table 3, which is better than the other methods that are used for runtime comparison.

Table 3. Accuracy comparison of our proposed technique with others

\begin{tabular}{cc}
\hline Author & Classification Accuracy (\%) \\
\hline H Ohmaid et al. (Our proposed) & 95 \\
MH Hamd and al. [23] & 94 \\
SG Firake and al. [24] & 92.85 \\
J poonia and al. [25] & 82.5 \\
\hline
\end{tabular}

\section{CONCLUSIONS}

In this paper, segmentation was achieved using pretreatment step based on an unsupervised neural approach. The normalization enabled the transformation of the segmented circular iris region into a fixed-size rectangular shape using Daugman's rubber sheet model. Discrete wavelet transformation (DWT) is used for extracting the optimum features of iris images and reducing the runtime of classification of these iris templates. Finally, a comparison was made between the classifiers SVM and KNN. Experimental evaluation using the URIBIS database clearly demonstrated the proposed technique performs in an efficient manner, especially for the KNN classifier that gave an accuracy of $95 \%$. 


\section{REFERENCES}

[1] Fang, Bin Et Tang, Yuan Yan. Improved class statistics estimation for sparse data problems in offline signature verification. IEEE Transactions on Systems, Man, and Cybernetics, Part C (Applications and Reviews). vol. 35, no 3, pp. 276-286, 2005.

[2] Gao, Xinbo, Zhong, Juanjuan, LI, Jie, et al. Face sketch synthesis algorithm based on E-HMM and selective ensemble. IEEE Transactions on Circuits and Systems for Video Technology, vol. 18, no 4, p. 487-496, 2008.

[3] Yu, Li, Zhang, David, Et Wang, Kuanquan. The relative distance of key point based iris recognition. Pattern Recognition, vol. 40, no 2, p. 423-430, 2007.

[4] Zhang, Taiping, Fang, Bin, Liu, Weining, et al. Total variation norm-based nonnegative matrix factorization for identifying discriminant representation of image patterns. Neurocomputing, vol. 71, no 10, p. 1824-1831, 2008.

[5] Daugman, J. Biometric personal identification system based on iris analysis, United States Patent, US 5291560. 1994.

[6] Daugman, J., "How iris recognition works," Image Processing. 2002. Proceedings. 2002 International Conference on, vol.1, no., pp.I-33, I-36 vol.1, 2002.

[7] Masek, Libor, et al. Recognition of human iris patterns for biometric identification. 2003.

[8] Ohmaid, Hicham, Eddarouich, S., Bourouhou, A., et al. "Iris segmentation using a new unsupervised neural approach". Int J Artif Intell (IJAI), vol. 9, no 1, pp.58-64, 2020.

[9] H. Ohmaid, M. Timouyas and S. Eddarouich, "A comparative study of similarity measurement in a new neural model of Unsupervised clustering," 2019 Third International Conference on Intelligent Computing in Data Sciences (ICDS), Marrakech, Morocco, 2019, pp. 1-8, doi: 10.1109/ICDS47004.2019.8942242.

[10] Timouyas M., Eddarouich S. and Hammouch A. "Mode region detection using improved Competitive Hebbian Learning for unsupervised clustering," Engineering Science and Technology: An International Journal (ESTIJ), ISSN: 2250-3498 Vol.7, No.4, pp. 26-35, 2017.

[11] Parzen, E. "An Estimation of a Probability Density Function and Mode," Ann. Math. Stat., vol. 33, pp.1065-1076, 1962.

[12] Postaire, J.-G., \& Vasseur, C. P. A. "A fast Algorithm for non Parametric Probability Density Estimation," IEEE, Trans. on Pattern Anal. and Machine Intel. PAMI-4, n 6, pp. 663-666, 1982.

[13] Eddarouich, S., \& Sbihi, A. "Neural Network for Modes Detection in Pattern Classification.” ICTIS'07, Morocco, Fez, 3-5 pp. 300-303, 2007.

[14] Timouyas, M., Eddarouich, S., and Hammouch, A. "A new approach of classification for non-Gaussian distribution upon competitive training," (ICCS'12), Agadir, Morocco, pp.1-6, 2012.

[15] Eddarouich, S., and Sbihi, A. "Détection des Modes par Approche Neuronale pour la Classification des Données d'un Mélange des Distributions Normales," Proceeding of the 6th African Conference on Research in Computer Science (CARI'02), Yaoundé, Cameroon, pp. 61-68, 2002.

[16] Wildes, Richard P. Iris recognition: an emerging biometric technology. Proceedings of the IEEE, vol. 85, no 9, p. 1348-1363, 1997.

[17] Ma, Li, Tan, Tieniu, Wang, Yunhong, et al. Efficient iris recognition by characterizing key local variations. IEEE Transactions on Image processing, vol. 13, no 6, p. 739-750, 2004.

[18] Ganorkar, Sanjay Et Memane, Mayuri. "Iris recognition using discrete wavelet Transform." International Journal of Advances in Engineering \& Technology, vol. 4, no 1, p. 356, 2012.

[19] Rana, Humayan Kabir, Azam, Md Shafiul, Akhtar, Mst Rashida, et al. "A fast iris recognition system through optimum feature extraction". Peer J Computer Science, vol. 5, p. e184, 2019.

[20] Czajka, A., Bowyer, K. W., Krumdick, M., \& VidalMata, R. G. "Recognition of image-orientation-based iris spoofing". IEEE Transactions on information Forensics and Security, 12(9), 2184-2196, 2017.

[21] Zhang, Hui and Guan, Xiangfeng. Iris recognition based on grouping KNN and rectangle conversion. In: 2012 IEEE International Conference on Computer Science and Automation Engineering. IEEE. p. 131-134, 2012.

[22] “Ubiris: Noisy Visible Wavelength Iris Image Databases.” [Online]. Available: URL:http://iris.di.ubi.pt/.

[23] Hamd, Muthana H. and Ahmed, Samah K. Biometric system design for iris recognition using intelligent algorithms. International Journal of Modern Education and Computer Science, vol. 11, no 3, p. 9, 2018.

[24] Firake, Sarita G. and Mahajan, P. M. Comparison of iris recognition using gabor wavelet, principal component analysis and independent component analysis. International Journal of Innovative Research in Computer and Communication Engineering, vol. 4, no 6, p. 12334-12342, 2016.

[25] Jyoti, P., Parvati, B., Sandeep, K. G., and al. New improved feature extraction approach of IRIS recognition. International Journal of Computer Systems, vol. 3, no 1, p. 1-3, 2016. 